\title{
Nanooptics of Plasmonic Nanomatryoshkas: Shrinking the Size of a Core-Shell Junction to Subnanometer
}

\author{
Li Lin, ${ }^{\dagger}$ Mario Zapata, ${ }^{\ddagger}, \S$ Min Xiong, ${ }^{\dagger}$ Zhonghui Liu, $^{\dagger}$ Shanshan Wang, ${ }^{\dagger}$ Hong Xu, ${ }^{\dagger}$ Andrei G. Borisov, $"$ \\ Hongchen $\mathrm{Gu}^{\dagger}$ Peter Nordlander, ${ }^{*}, \perp$ Javier Aizpurua, ${ }^{*,+}$ and Jian $\mathrm{Ye}^{*, \dagger}$ \\ ${ }^{\dagger}$ School of Biomedical Engineering and Med-X Research Institute, Shanghai Jiao Tong University, 1954 Huashan Road, Shanghai, \\ 200030, China \\ ${ }^{\ddagger}$ Material Physics Center CSIC-UPV/EHU and Donostia International Physics Center DIPC, Paseo Manuel de Lardizabal 5, \\ Donostia-San Sebastián, Spain \\ ${ }^{\S}$ Departamento de Física, Universidad de los Andes, Bogotá, Colombia \\ "Institut des Sciences Moléculaires d'Orsay, , UMR 8214 CNRS-Université Paris-Sud, Bâtiment 351, 91405 Orsay Cedex, France \\ ${ }^{\perp}$ Department of Physics and Astronomy, MS 61, Rice University, Houston, Texas 77005, United States
}

\section{Supporting Information}

ABSTRACT: Quantum effects in plasmonic systems play an important role in defining the optical response of structures with subnanometer gaps. Electron tunneling across the gaps can occur, altering both the far-field optical response and the near-field confinement and enhancement. In this study, we experimentally and theoretically investigate plasmon coupling in gold "nanomatryoshka" (NM) nanoparticles with different core-shell separations. Plasmon coupling effects between the core and the shell become significant when their separation decreases to $15 \mathrm{~nm}$. When their separation decreases to below $1 \mathrm{~nm}$, the near- and far-field properties can no longer be described by classical approaches but require the inclusion of quantum mechanical effects such as electron transport through the self-assembled monolayer of molecular junction. In addition, surface-enhanced Raman scattering measurements indicate strong electron-transport induced charge transfer across the molecular junction. Our quantum modeling provides an estimate for the AC conductances of molecules in the junction. The insights acquired from this work pave the way for the development of novel quantum plasmonic devices and substrates for surface-enhanced Raman scattering.

KEYWORDS: Plasmon, nanoshells, quantum plasmonics, tunneling plasmon

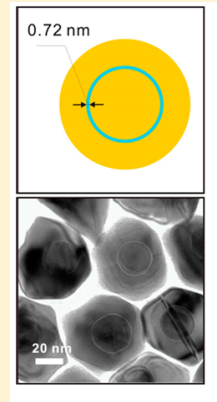

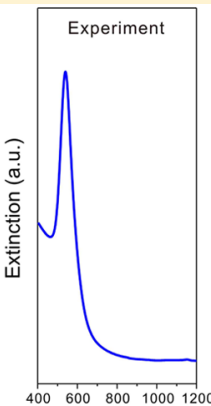

$600 \quad 8001000120$ Wavelength $(\mathrm{nm})$

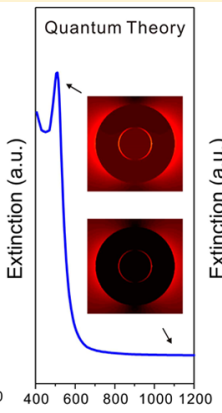

6008001000120040060080010001200 Wavelength $(\mathrm{nm})$ Wavelength $(\mathrm{nm})$
$\mathrm{T}^{\mathrm{s}}$ he optical excitation of collective oscillations of electrons on the surfaces of metallic nanostructures results in the well-known surface plasmon resonances. One of the most interesting properties of plasmon resonances is their associated near-field enhancement and confinement, which overcomes the diffraction limit of conventional optics and exhibits great potential in numerous areas including energy harvesting, ${ }_{5,2}^{1,2}$ material science, ${ }^{3,4}$ information communication, ${ }^{5,6}$ and life sciences. ${ }^{7,8}$ The cavity mode formed in a narrow nanogap is determined by the coupling of neighboring nanostructures and causes this near-field confinement to be particularly pronounced as well as highly sensitive to the dielectric environment. ${ }^{9-12}$

Recently, there have been increasing interests in the experimental and theoretical investigations of plasmonic "nanomatryoshka" (NM) particles, ${ }^{13-23}$ which consists of a spherical metallic core surrounded by a concentric metallic shell separated by a dielectric layer (see Figure 1A), due to its significant potential in multiplexed biosensing and bioimaging application. $^{24,25}$ The interior "nanogaps", that is, the core-shell separation, can be easily tuned by controlling the thickness of the dielectric spacer, consequently controlling the extent of the coupling and induced near-field enhancement between the core and shell. This phenomenon has been well explained by classical electromagnetic theory, which predicts strong electric field enhancements in the nanogaps region. ${ }^{13,14,20,21}$ However, when junction gaps reach subnanometer separation distances, recent studies have shown that quantum mechanical effects, related to tunneling and nonlocal screening, may play an increasingly important role and lead to a reduction in the electric field enhancements. $^{26-29}$ Because classical electromagnetic theory fails to describe plasmonic behavior in such subnanometer gaps, different models have been developed to account for electron tunneling across gaps. ${ }^{30-35}$ For a NM with core-shell separations below $0.4 \mathrm{~nm}$, calculations from time-dependent

Received: April 27, 2015

Revised: September 12, 2015

Published: September 16, 2015 


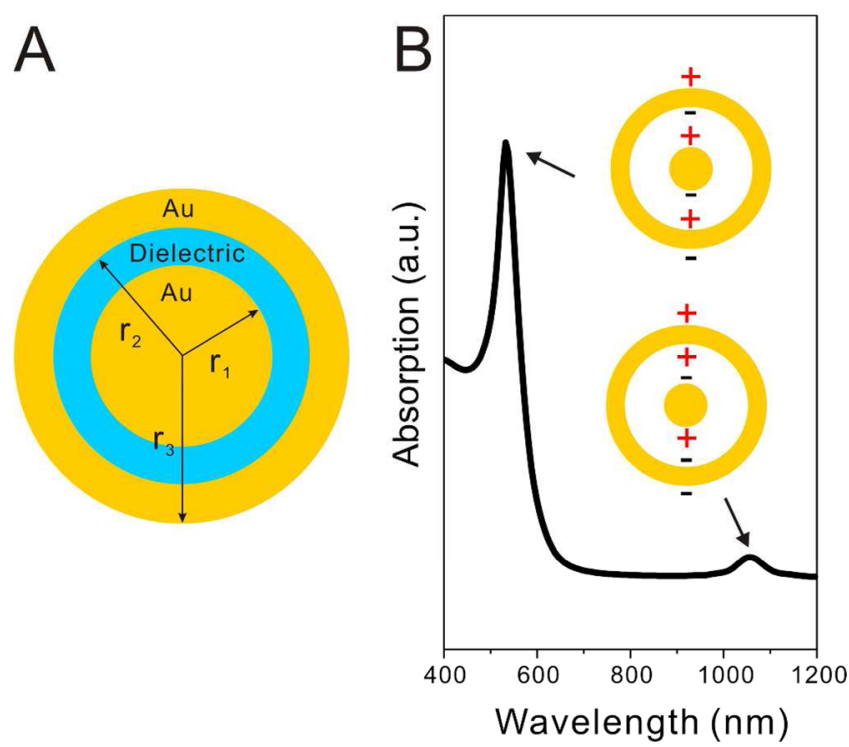

Figure 1. (A) Geometrical illustration of a nanomatryoshka (NM). (B) Absorption cross-section calculated using classical electromagnetic theory for a $\mathrm{NM}\left(r_{1}, r_{2}, r_{3}\right)=(12.5,13.5,30) \mathrm{nm}$, with a $\mathrm{SiO}_{2}$ spacer layer. Insets represent the schematic surface charge distributions for the modes at $532 \mathrm{~nm}$ (left) and $1057 \mathrm{~nm}$ (right).

density functional theory (TDDFT) have predicted pronounced tunneling across the nanogap, which significantly modifies the absorption cross-section and the local electric field enhancements. ${ }^{22}$ More importantly, advances in the synthesis of NMs with precise and uniform subnanometer gaps provide a better platform for experimental investigation of quantum plasmonic effects, $^{17,25,36,37}$ compared to typical nanoparticle dimer structures fabricated by various state-of-the-art techniques including high-resolution electron-beam lithography, ${ }^{30}$ electron-beam induced manipulation, ${ }^{26}$ dual AFM tip approaching, ${ }^{28}$ and dropcasting. ${ }^{27,31}$

In this Letter, we present a comprehensive investigation of the optical properties of NMs with different nanogap widths ranging from $100 \mathrm{~nm}$ down to the subnanometer regime. The larger gaps are realized by controlling the thickness of $\mathrm{S} \mathrm{SiO}_{2}$ layer inside the gap, while the subnanometer gap is created using a selfassembled monolayer (SAM) of 1,4-benzenedithiol (1,4-BDT) molecules as well as of 4-methylbenzenethiol (4-MBT) molecules. The measured far-field optical spectra of these structures are compared with simulations from the classical electromagnetic theory and from the quantum-corrected model (QCM). ${ }^{32}$ The QCM is a theoretical approach that accounts for electron tunneling across the gap by replacing the junction with an effective medium characterized by the tunneling conductance. $^{32,38}$ We show that classical electromagnetic theory correctly predicts the optical properties of NMs with coreshell separations larger than a nanometer but fails for NMs with subnanometer sized gaps. This is because it neglects electron transport between the core and the shell through the selfassembled monolayer. In contrast, the QCM well describes both the far- and near-field optical properties in the subnanometer regime.

Figure 1A shows the geometry of a $\mathrm{Au}$ NM with the structural parameters $\left(r_{1}, r_{2}, r_{3}\right)$ representing the radius of the inner Au core and the inner and outer radii of the Au shell. In most of the present work, the dielectric spacer between the core and the shell will be either a $\mathrm{SiO}_{2}$ layer or a monolayer of 1,4-BDT molecules (the case of the 4-MBT molecules will be quoted specifically). Previous results have indicated that classical electromagnetic
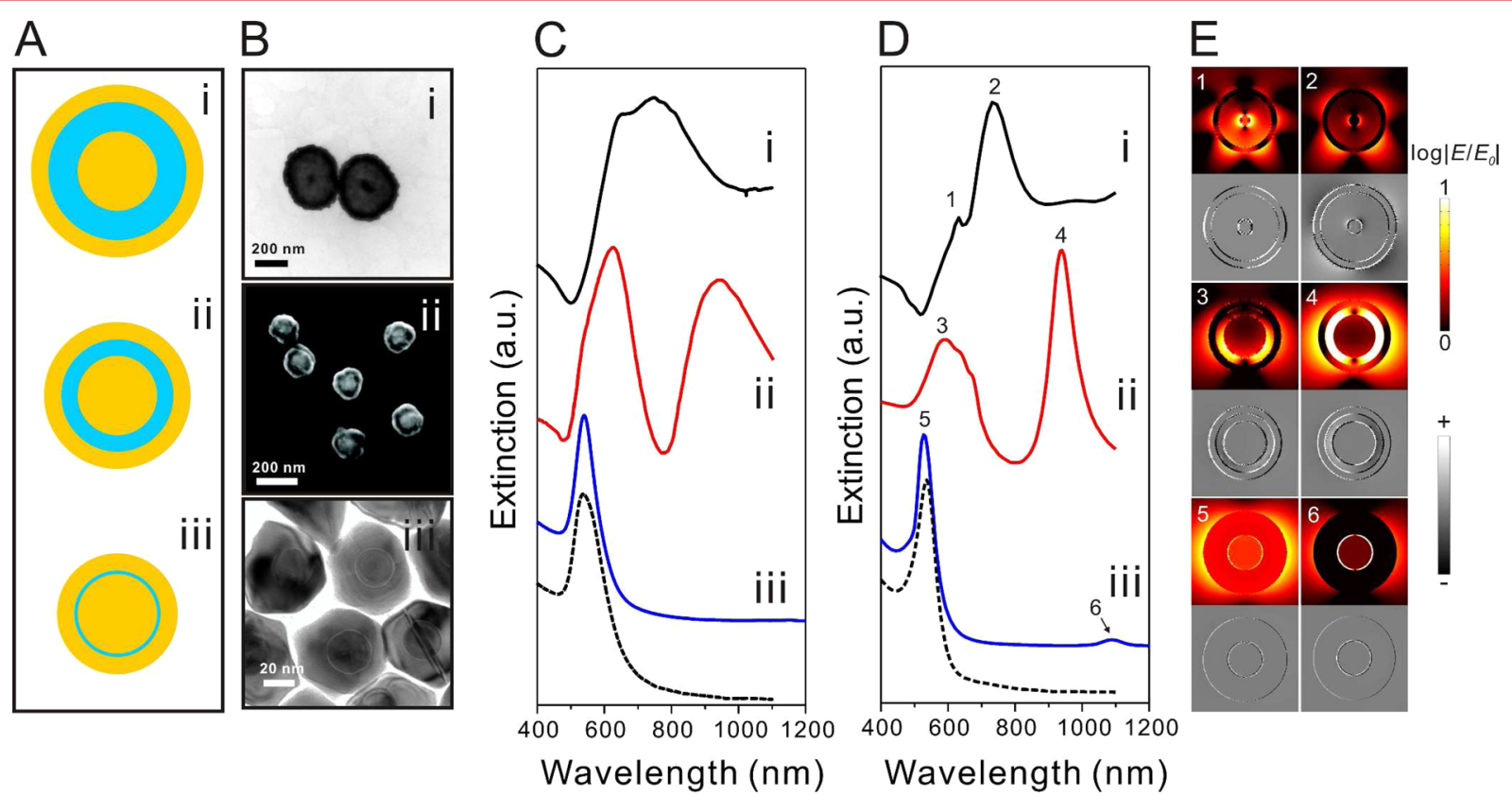

Figure 2. Optical properties of NMs of different geometries: (i) $(25,125,150) \mathrm{nm}$ with a gap size of $100 \mathrm{~nm}$ and with $\mathrm{SiO}_{2}$ space layer; (ii) $(40,55,68)$ $\mathrm{nm}$ with a gap size of $15 \mathrm{~nm}$ and with $\mathrm{SiO}_{2}$ spacer layer; (iii) $(10,10.7,25) \mathrm{nm}$ with a gap size of $0.7 \mathrm{~nm}$ and with 1,4-BDT spacer layer. (A) Geometrical illustration, (B) TEM/SEM images, (C) experimentally measured extinction spectra, and (D) calculated extinction spectra using classical electromagnetic theory for the NMs (i-iii). Dashed lines in (C,D) show the experimentally measured and calculated extinction spectra of aqueous Au nanoparticles with a radius of $25 \mathrm{~nm}$, respectively. (E) Simulated electric field intensity $\left(\log \left|E / E_{0}\right|\right.$ ) distributions (top) and surface charge distributions (bottom) at resonance corresponding to the modes 1-6, using classical electromagnetic theory. The SEM image in (B) and the measured extinction spectrum in (C) for the NM (ii) were reproduced from ref 37 for comparison between the NMs with different gap sizes. 
theory is appropriate for describing the optical response of NMs with gaps equal or larger than $1 \mathrm{~nm} .{ }^{22}$ Figure $1 \mathrm{~B}$ displays the absorption spectrum of a NM of geometry $(12.5,13.5,30) \mathrm{nm}$ calculated using classical electromagnetic theory. The calculations were performed using a finite difference time domain (FDTD) method to solve the electromagnetic Maxwell equations with the program of FDTD Solutions (Lumerical Solutions, Inc., Canada). The empirical dielectric functions of $\mathrm{Au}$ and $\mathrm{SiO}_{2}$ were fitted by Lumerical's multicoefficient model (MCM). ${ }^{39,40}$ The refractive index of molecular SAMs is assumed 1.4. ${ }^{41}$ The simulation mesh size was set as $0.1 \mathrm{~nm}$ and the whole simulation region is defined with a background index of 1.33 corresponding to water. The calculated absorption spectrum shows a strong resonance at $532 \mathrm{~nm}$ and a relatively weak resonance at $1057 \mathrm{~nm}$, which can be explained within the framework of plasmon hybridization. ${ }^{13}$ The resonance at 1057 $\mathrm{nm}$ corresponds to the excitation of the bonding hybridized plasmon of NM formed by the bonding dipolar plasmon mode of the shell with admixture of the dipolar plasmon mode of the core. The resonance at $532 \mathrm{~nm}$ can be assigned to a hybridized plasmon mode of the NM formed from the dipolar mode of the core and the antibonding mode of the shell. The insets of Figure 1B illustrate the surface charge distributions of the two resonances. Computations based on the quantum mechanical modeling have predicted that tunneling occurs at around $0.4 \mathrm{~nm}$ size for a vacuum gap and the mode at $1057 \mathrm{~nm}$ is highly sensitive to quantum effects due to the high surface charge density and the right charge symmetry in the nanogap. ${ }^{22,23}$ We experimentally explore this situation in this letter.

In order to investigate the plasmon hybridization and the importance of quantum effects in the NMs, we have developed a wet-chemistry synthesis technique to prepare $\mathrm{Au} \mathrm{NMs}$ with different gap sizes. Figure 2A shows the schematic illustration of NMs with gaps in three size ranges: (i) $\sim 100 \mathrm{~nm}$, (ii) $\sim 10 \mathrm{~nm}$, and (iii) sub-1 $\mathrm{nm}$. The nanogap is the primary parameter influencing the plasmon coupling effects between the core and the shell. For example, a $100 \mathrm{~nm}$ gap size may only induce a weak plasmon coupling because the decay length of near-fields normal to the metal surface typically extends only approximately $10-20$ nm. ${ }^{42}$ In contrast, an $\sim 10 \mathrm{~nm}$ gap size causes a strong coupling, hybridization, and near-field enhancement. When the gap size reaches the subnanometer scale, electron transport between the core and the shell may occur and can influence both the near- and far-field optical properties.

NMs with dimensional parameters $(25,125,150) \mathrm{nm}(\mathrm{i}$, Figure 2B) were synthesized using a procedure reported elsewhere. ${ }^{43}$ Briefly, $50 \mathrm{~nm}$-sized citrate Au NPs were prepared by adding sodium citrate into hydrogen tetrachloroaurate trihydrate $\left(\mathrm{HAuCl}_{4}\right)$ aqueous solution. $\mathrm{A} \mathrm{SiO}_{2}$ layer was coated around the Au NPs by a Stöber process with the reaction of ammonia and tegraethyl orthosilicate (TEOS) in 2-propanol. The outer Au shell was formed using a chemical plating method, which is typically used in the fabrication of conventional $\mathrm{Au}$ nanoshells. ${ }^{44}$ TEM image in Figure 2B (JEOL-2010, JEOL) confirms the presence of the $\mathrm{SiO}_{2}$ layer thickness of $100 \mathrm{~nm}$ and the Au shell thickness of $25 \mathrm{~nm}$ in average. The redshift of $20 \mathrm{~nm}$ in the extinction spectrum also verifies the presence of the coating of $\mathrm{SiO}_{2}$ layer on the Au cores (see i and ii in Figure S1A in Supporting Information). The measured extinction spectrum (Lambda 950, PerkinElmer) of the obtained NMs (i, Figure 2C) shows two broad resonance peaks at 656 and $747 \mathrm{~nm}$, respectively. The FDTD calculated extinction spectrum (i, Figure 2D) agrees well with the experimental result, indicating that the two peaks correspond to the hexapolar resonance of the outer Au shell for mode 1 and to a quadrupolar resonance for mode 2 , as obtained from the calculated electric field distributions and surface charge profiles (top panels in Figure $2 \mathrm{E}$ ). An additional intense dipolar resonance mode appears beyond $1200 \mathrm{~nm}$ according to our calculations (data not shown). The strong coupling between these higher-order modes (mode 1 and 2) of the NMs to light is primarily attributed to phaseretardation effects due to their relatively large particle size. ${ }^{45}$ The weak electric field enhancements in the gap region imply a rather weak plasmon coupling between the core and the shell, and therefore the far-field optical spectrum of NMs $(25,125,150)$ $\mathrm{nm}$ is here dominated by the scattering spectrum of the outer $\mathrm{Au}$ shell.

By optimizing the Stöber process, the $\mathrm{SiO}_{2}$ layer in the gap of the NMs can be shrunk to the tens of nanometers range. $14,15,20,46$ An example is the NMs of geometry $(40,55,68) \mathrm{nm}$ (gap size of $15 \mathrm{~nm}$ ) from ref 14 (ii, Figure 2B). Their experimental extinction spectrum shows two intense and broad peaks at 622 and $943 \mathrm{~nm}$ (ii, Figure 2C). The corresponding FDTD calculated extinction spectrum reproduces the experimental features well with two resonances at 592 and $940 \mathrm{~nm}$, denoted as mode 3 and 4 in Figure 2D. Mode 3 is the bonding mode resulting from the dipolar core and bonding shell modes, and mode 4 is the bonding mode arising from the core and the antibonding shell dipolar modes, as confirmed by the calculated surface charge distributions (see corresponding panels in Figure 2E). For mode 4, the pronounced electric field enhancements also suggest a significant coupling between the core and the shell plasmons due to the relatively small gap size. This mode coupling may also result in a Fano resonance in the optical response of NMs. ${ }^{20}$ In addition, we note that the line width of the experimentally measured plasmon resonances is broader than those of the calculated ones, which is most likely due to the inhomogeneous gap size and the polydispersed particle size of the synthesized NMs.

A recent advance in the synthesis method for shell-isolated nanoparticles enables the growth of as thin $\mathrm{SiO}_{2}$ layers as $2 \mathrm{~nm}$. ${ }^{47}$ However, to further reduce the NMs' core-shell below $2 \mathrm{~nm}$ remains a challenge. In order to obtain the NMs with subnanometer gaps, we instead use a self-assembled monolayer of 1,4-BDT molecules as a spacer to uniformly and reproducibly control the core-shell separation. A TEM image of NMs with a geometry of $(10,10.7,25) \mathrm{nm}$ is shown in Figure 2B (iii). The core-shell separation was calculated as $0.72 \mathrm{~nm}$ on average, based on the high-resolution TEM measurements on 260 sites of 65 nanoparticles in order to reach a statistically significant sample size (Figure 3). Such NMs were synthesized in a layer-by-layer process, starting with the preparation of $20 \mathrm{~nm}$ diameter $\mathrm{Au}$ cores. ${ }^{37}$ A self-assembled monolayer of 1,4-BDT molecules was formed to replace the original capping agent of cetyltrimethylammonium chloride (CTAC) on the surface of Au cores. After the removal of the excess 1,4-BDT molecules, a Au shell of desired thickness is grown on the cores by adding $\mathrm{HAuCl}_{4}$, CTAC (as a capping agent) and ascorbic acid (as a mild reducing agent). The successful formation of a monolayer of 1,4-BDT molecules on the Au cores was also confirmed by the $3 \mathrm{~nm}$ redshift of the extinction spectrum after the replacing process (see Figure S1B in Supporting Information). Because both ends of 1,4-BDT molecules covalently connect to the core and shell surface via the $\mathrm{Au}-\mathrm{S}$ bond, the molecular length determines the core-shell separation. Assuming that 1,4-BDT has a perpendicular orientation relative to the Au surfaces inside the gap, the gap 
A
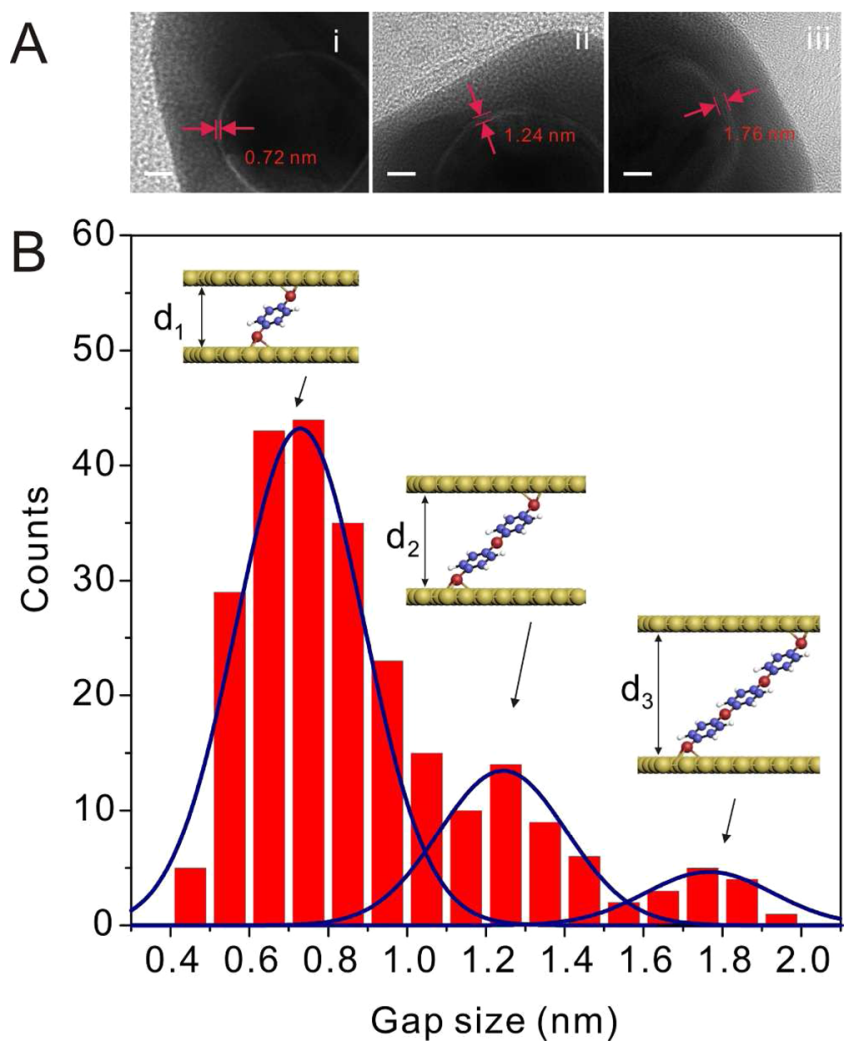

Figure 3. (A) High-resolution TEM images and (B) histogram of different gap sizes in NMs. All scale bars correspond to $5 \mathrm{~nm}$. For panel $\mathrm{B}$, the three gap-size peaks fitted by Gaussian model correspond to i-iii in panel A and are centered at (i) $0.72 \mathrm{~nm}$; (ii) $1.24 \mathrm{~nm}$; (iii) $1.76 \mathrm{~nm}$. The insets in panel $\mathrm{B}$ illustrate the schematic hypothetical configurations of 1,4-BDT molecules inside the gaps.

size is estimated to be the thickness of 1,4-BDT monolayer, that is, in the range of 0.8 to $1 \mathrm{~nm}$, using the known bond lengths, bond angles, and the approximate distance between the sulfur atom and the gold surface of the calculations by density functional theory. ${ }^{48-50}$ However, different gap sizes were also observed in high-resolution TEM images (Figure 3A) and thus a series of gap sizes were carefully measured to obtain structural information on the gap through the corresponding histogram (Figure 3B). The first dominant peak in the gap size distribution is centered at a gap size of $0.72 \mathrm{~nm}$, which likely corresponds to a slightly tilted 1,4-BDT configuration with an angle of approximately $25^{\circ}-45^{\circ}$ with respect to the surface normal for low surface coverage. Such a tilt is in agreement with previous estimates and calculations. ${ }^{27,49,50}$ The second and third peaks in the histogram are centered at gap separations of 1.24 and 1.76 $\mathrm{nm}$, which most likely corresponds to a bilayer and a trilayer of 1,4-BDT molecules, respectively (see insets in Figure 3B). ${ }^{50}$ In addition to previous estimates by ellipsometry and calculations, TEM imaging of various gap sizes in NMs offers a direct observation of different thicknesses of 1,4-BDT molecular films on a Au surface. It has been noted that a $0.62 \mathrm{~nm}$ gap in NMs was reported previously and the authors had attributed the shrinkage of the 1,4-BDT monolayer to the compression of the molecules between the core and shell, proved by the blueshifts (shift to higher wavenumbers) of the phenyl ring stretching mode and the $\mathrm{CH}$ bending mode in Raman spectra. ${ }^{37}$ However, this blueshift of Raman bands was not observed in our measurement, a situation that can possibly be explained by the slight inclined configuration of 1,4-BDT molecules in our core-shell junctions instead of a compressed configuration. In contrast, we have observed a redshift of the Raman bands which will be explained below.

Because of their relatively small size, the extinction spectrum of such NMs is primarily dominated by the absorption cross-section (see Figure S2 in Supporting Information). We first simulated the extinction spectrum of a $(10,10.7,25) \mathrm{nm}$ NM with classical electromagnetic theory using FDTD. The results show a noticeable resonance at $526 \mathrm{~nm}$ (mode 5) and a weak resonance at $1090 \mathrm{~nm}$ (mode 6) in Figure 2D, which seem to behave similarly to the plasmon hybridizations of mode 3 and 4, due to identical surface charge patterns and near-field enhancements (bottom panels in Figure 2E). In contrast to the larger (40, 55, 68) $\mathrm{nm} \mathrm{NM}$, the narrower gap leads to stronger plasmon hybridization and energy level splitting, inducing a spectral redshift for the low energy mode and a blueshift to the high energy mode. However, the experimental extinction spectrum (iii, Figure 2C) shows only one pronounced resonance at 539 $\mathrm{nm}$, which is in direct contrast with the prediction by classical theory in Figure 2D (iii).

Quantum mechanical modeling has predicted the disappearance of the lower energy mode (mode 6) as a direct manifestation of the dynamical screening of the mode due to quantum tunneling in gaps below $0.4 \mathrm{~nm}$. ${ }^{22}$ However, the coreshell-gap of the present system is too wide for quantum tunneling to occur. Indeed, in the present system the tunneling is assisted by the self-assembled molecular monolayer in the plasmonic gap, where the 1,4-BDT molecules play the role of molecular wires bridging the $\mathrm{Au}$ electrodes (core and shell) as has been extensively studied theoretically and experimentally in the context of molecular electronics. ${ }^{51-61}$ 1,4-BDT has two thiol groups that form covalent bonds to $\mathrm{Au}$, therefore creating a conductive link through the $\pi$-orbitals of the phenyl ring due to a strong overlap with the Au electrons. ${ }^{62}$ Recent studies have demonstrated that, indeed, the optical response of plasmonic systems can be affected by electron tunneling through molecular monolayer (s) bridging narrow gaps between the particles. ${ }^{27,63}$ Similar to the metal-to-metal tunnel junction, the tunneling current through the molecular tunnel junction short circuits the junction, neutralizes the plasmon induced charges across the gap, and leads to the disappearance of the corresponding plasmonic mode from the optical spectra. To support this idea, we show the measured and calculated extinction spectra for homogeneous $\mathrm{Au}$ NPs with the same outer diameter as the NM $(50 \mathrm{~nm})$ (dashed lines in Figure 2C,D). The measured spectrum of these solid particles agree almost perfectly with that of 1,4-BDT embedded NMs as if the gap were absent, which points at the presence of tunneling through the gap in the $(10,10.7,25) \mathrm{nm}$ NMs.

To model the effect of quantum tunneling on the optical properties of the NM, we use the QCM approach ${ }^{28,32}$ modified to account for electron transport through the molecular layer. Briefly, we model the 1,4-BDT SAM using a permittivity: ${ }^{63,64}$

$$
\varepsilon_{\mathrm{SAM}}=\varepsilon_{\infty}+i \frac{4 \pi \sigma}{\omega}
$$

where $\varepsilon_{\infty}=2 .^{41}$ Assuming a linear optical potential variation across the gap, the conductivity $\sigma$ of the molecular layer is given by $\sigma=\alpha G_{0}(d / \Sigma)$. Here $G_{0}=7.748 \times 10^{-5} \mathrm{~S}$ is the quantum of conductance, $d=r_{2}-r_{1}$ is the width of the gap, and $\Sigma=0.22 \mathrm{~nm}^{2}$ is the typical surface area per molecule in a dense SAM. ${ }^{65}$ The coefficient $\alpha$ gives the conductance of the single molecule $G$ in units of $G_{0}: G=\alpha G_{0}$. Figure 4A shows the extinction spectra of a $(10,10.7,25) \mathrm{nm}$ NM calculated both using QCM and a 
A

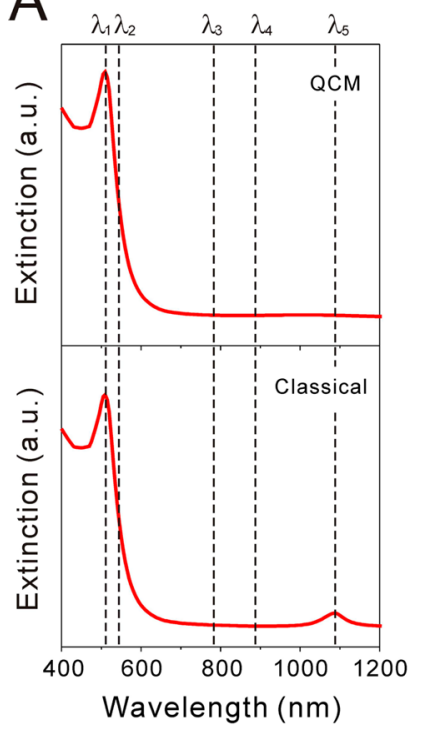

\section{$\mathrm{C}$}

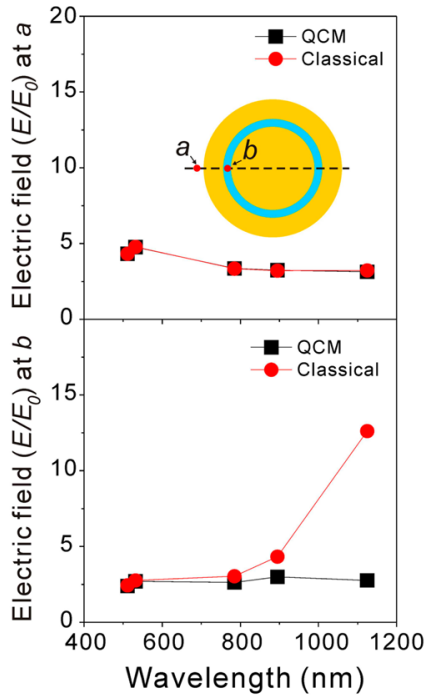

D

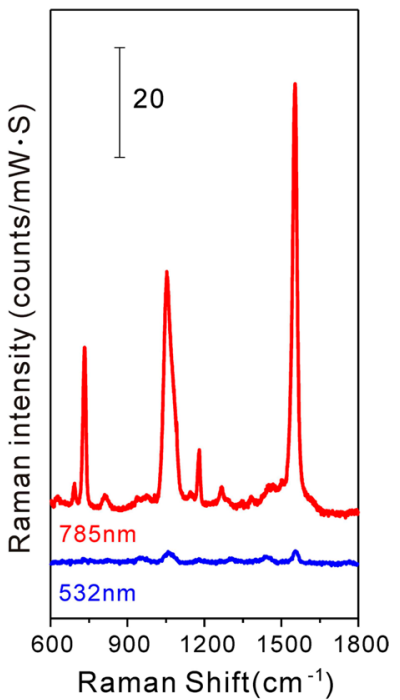

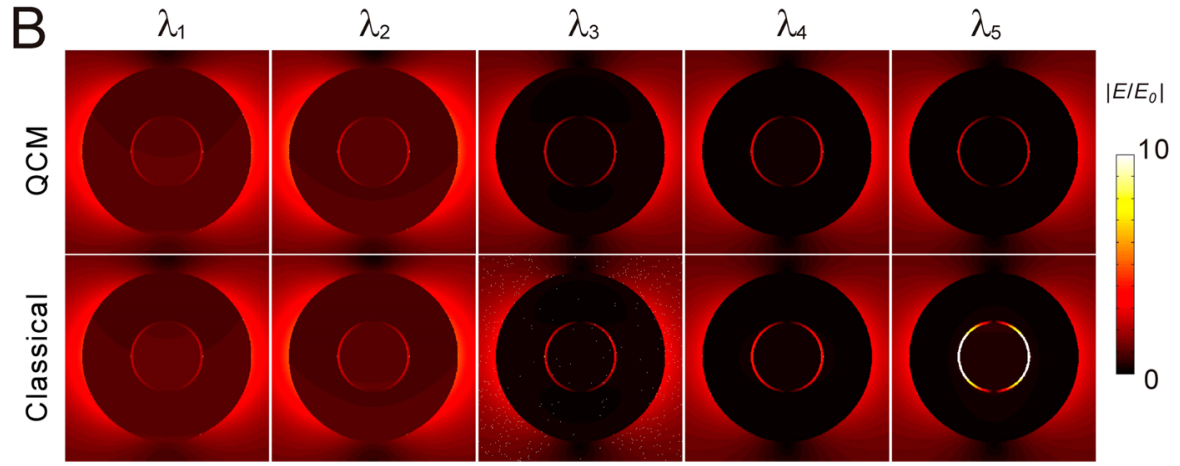

Figure 4. (A) Extinction spectra, (B) electric field distributions, and (C) electric field intensities at point $a$ and $b$ for a NM $(10,10.7,25) \mathrm{nm}$ calculated by QCM using conductive molecule junction $\left(0.08 G_{0}\right.$ per molecule $)$ and classical theory. Electric fields were calculated at the wavelengths of $\lambda_{1}=511 \mathrm{~nm}$, $\lambda_{2}=532 \mathrm{~nm}, \lambda_{3}=785 \mathrm{~nm}, \lambda_{4}=895 \mathrm{~nm}, \lambda_{5}=1100 \mathrm{~nm}$. Inset in panel C indicates the position of point $a(0.1 \mathrm{~nm}$ apart from the outer surface of Au shell $)$ and $b(0.1 \mathrm{~nm}$ apart from the surface of Au core). (D) SERS spectra of NMs $(10,10.7,25) \mathrm{nm}$ with 1,4-BDT spacer layer excited by 532 and $785 \mathrm{~nm}$ laser.

standard classical approach, the boundary element method (BEM). ${ }^{66}$ In QCM calculations, we use $\alpha=0.08$, which is the lower limit of the conductance per molecule at which the plasmonic mode at $\sim 1100 \mathrm{~nm}$ disappears from the optical spectrum (see also Figure 5). The figure shows excellent agreement between the QCM and measured spectrum with a strong dipolar mode around $520 \mathrm{~nm}$ but no sign of the longer wavelength hybridized mode that is otherwise present around $1100 \mathrm{~nm}$ in the classical approach. Several remarks are in order with respect to this result: (i) As we will also discuss in connection with Figure 5, $G=0.08 G_{0}$, provides a lower estimate for the conductance of the 1,4-BDT SAM needed to quench the plasmon resonance at $1100 \mathrm{~nm}$. Since $\sigma \sim \alpha / \Sigma$, less dense molecular layer than supposed here will require higher conductance per molecule to reach the same effect. (ii) The present value of the molecular conductance is within the range of the experimental and theoretical estimates for electron transport in individual $1,4-\mathrm{BDT}$ molecules, ${ }^{51-61}$ particularly taking into account that the inclined configuration of SAM tends to increase the conductance values. ${ }^{58-61}$ (iii) For laser-assisted transport in molecular junctions the ac conductance can be significantly higher that the dc conductance measured in break junctions, in particular when photon absorption opens new conductance channels through molecular orbitals. ${ }^{67-69}$
Figure 4B shows electric field distributions calculated by QCM and by the classical approach for wavelengths of 511, 532, 785, 895 , and $1100 \mathrm{~nm}$. The classical approach predicts a large field enhancement in the interior gap for the $1100 \mathrm{~nm}$ mode while no such field enhancement is present in the QCM where the tunneling current short-circuits the gap. The distribution of electric field maps indicates that within the framework of classical theory and QCM, the near-field enhancement on the outer shell surface reaches the maximum at the hybridized dipolar resonance at short wavelength $(532 \mathrm{~nm})$. This qualitative information from near field maps is further analyzed in Figure $4 \mathrm{C}$ where we plot the electric field enhancement at the local points $a$ and $b$ (see the inset in Figure 4C). For example, at point $a$ (out of the shell), both within the QCM or classical theory, the electric field enhancement maintains a value below 5 for all wavelengths with a maximum of 4.7 at $532 \mathrm{~nm}$, which is very similar to the response of a solid spherical Au nanoparticle. For point $b$ (within the gap), classical theory predicts that the field enhancement increases when the wavelength becomes longer with a value below 5 at 511, 532,785 , and $895 \mathrm{~nm}$ and a maximal value of $\sim 13$ at $1100 \mathrm{~nm}$, while QCM shows constant field enhancements for all wavelengths with a maximal value below 3 . Note the remarkably different near-field behavior in plasmonic gap (for point $b$ ) at longer wavelength, where the strong near-field enhancement 


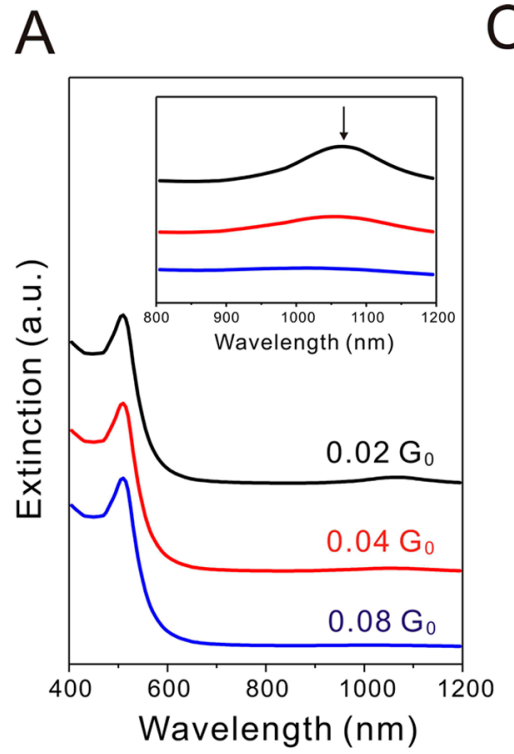

C
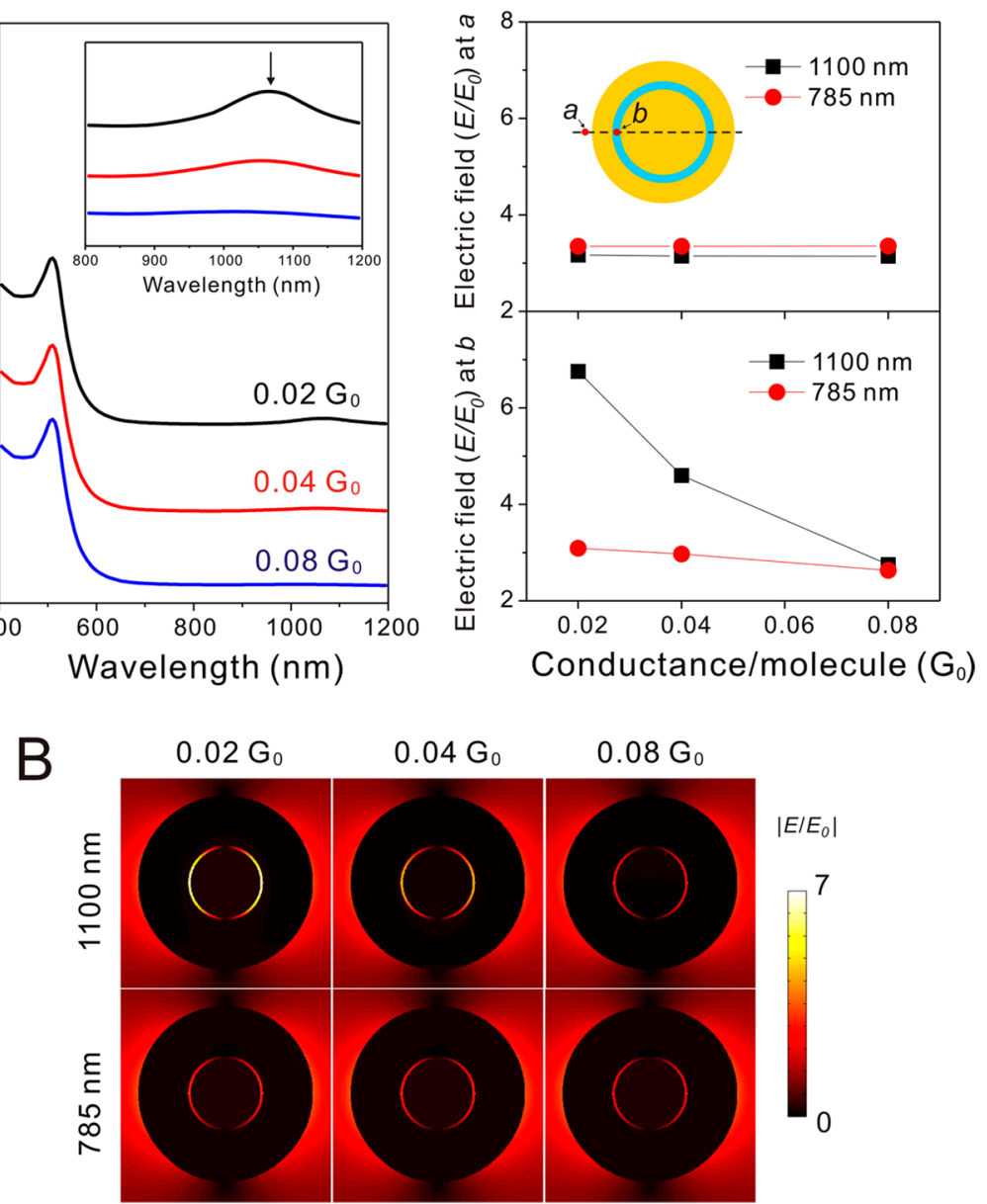

Figure 5. (A) Extinction spectra, (B) electric field distributions, and (C) electric field intensities at point $a$ and $b$ for a NM $(10,10.7,25) \mathrm{nm}$ calculated by QCM using conductive molecule junction with different conductances $\left(G_{0}\right)$. Inset in panel A is a zoom-in around high wavelength region. Electric fields were calculated at the wavelengths of 785 and $1100 \mathrm{~nm}$. Inset in panel C indicates the position of point $a(0.1 \mathrm{~nm}$ apart from the outer surface of Au shell) and $b(0.1 \mathrm{~nm}$ apart from the surface of Au core).
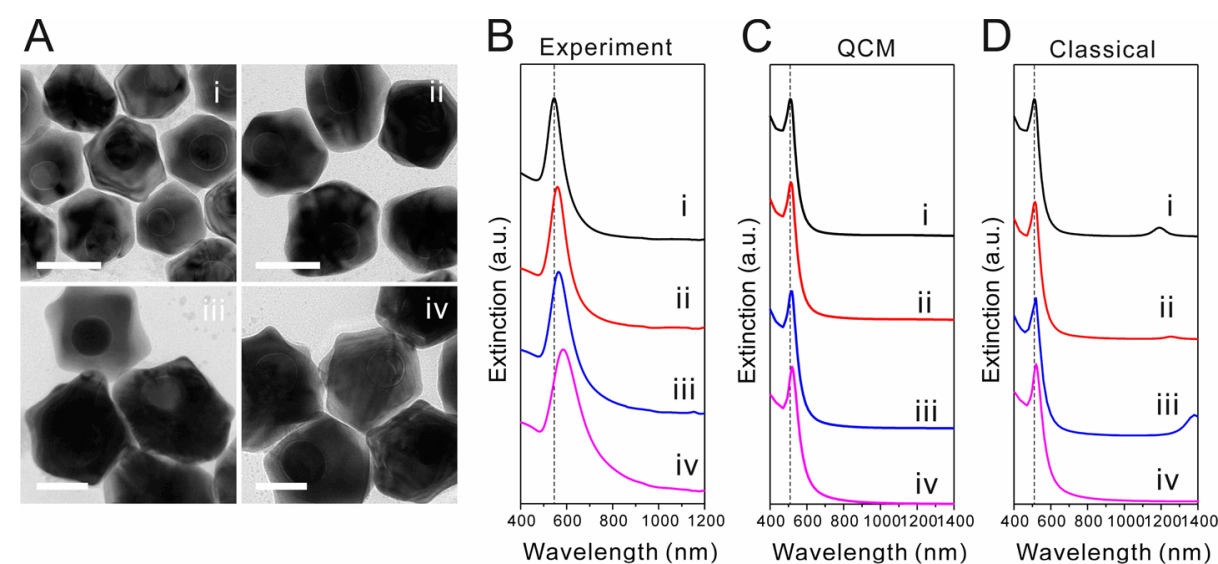

Figure 6. (A) TEM images, (B) experimentally measured and (C, D) calculated extinction spectra using (C) QCM and (D) classical electromagnetic theory for NMs (i-iv) with 1,4-BDT spacer layer and with different dimensions: (i) $(12.5,13.2,31) \mathrm{nm}$; (ii) $(14,14.7,37) \mathrm{nm}$; (iii) $(18,18.7,41.5) \mathrm{nm}$; (iv) $(21,21.7,50) \mathrm{nm}$.

calculated with classical theory is clearly suppressed in the description by the QCM.

In Figure 5A, we show the extinction spectra the NM (10, 10.7, 25) $\mathrm{nm}$ calculated by QCM as a function of junction conductance per 1,4-BDT molecule. As the conductance per molecule of the junction increases, the resonance at the longer wavelength gradually decreases and finally vanishes for the largest conductance of $0.08 G_{0}$, while the resonance at the visible range remains constant at around $520 \mathrm{~nm}$. Thus, we estimate $0.08 G_{0}$ as a lower limit for conductance per 1,4-BDT molecule needed to 
explain the quenching of the gap mode in the measured spectra. Figure 5B,C shows the corresponding NM's electric field maps and the electric fields at the local points at the junction (point $a$ ) and outer shell surface (point $b$ ) at the wavelengths of 785 and $1100 \mathrm{~nm}$ with different junction conductances (see the inset in Figure 5C). The wavelength of $785 \mathrm{~nm}$ was chosen because it is the excitation wavelength of the Raman measurements that will be discussed below. The results clearly indicate that the variation of junction conductance has much more dramatic impact on the near-field enhancement in the junction for the $1100 \mathrm{~nm}$ mode compared to the $785 \mathrm{~nm}$ mode; electric field enhancements decrease from $\sim 7$ to $\sim 2.5$ when the conductance increased from 0.02 to $0.08 G_{0}$. In contrast, the variation of junction conductance has almost no influence on the near-field enhancement at the outer shell surface. With QCM modeling, the strong dependence between the junction conductance and (near- and far-field) optical properties of the NM $(10,10.7,25) \mathrm{nm}$ can be satisfactorily explained by the short-circuiting of the gap. The modeling developed here also offers an approach to quantitatively estimate the specific molecular conductance across the gap based on the far-field optical spectral measurement.

We complete our discussion of the quenching of gap plasmons in 1,4-BDT embedded NMs, with a study of additional NMs of different geometries showing subnanometric gaps (Figure 6A): (i) $(12.5,13.2,31) \mathrm{nm}$; (ii) $(14,14.7,37) \mathrm{nm}$; (iii) $(18,18.7$, $41.5) \mathrm{nm}$; (iv) $(21,21.7,50) \mathrm{nm}$. In each of these structures, the core-shell separation is $0.72 \mathrm{~nm}$ corresponding to the molecular length of 1,4-BDT. All measured extinction spectra of NMs (i iv) display only one resonance peak in the visible regime (Figure $6 \mathrm{~B})$. The resonance peak redshifts from 544 to $585 \mathrm{~nm}$ as the size of the particle is increased from 62 to $100 \mathrm{~nm}$ thus behaving as a homogeneous spherical Au NP. The QCM extinction spectra (Figure 6C) are in excellent agreement with the measured spectra. In contrast, classical theory (Figure 6D) predicts additional longer wavelength modes (unphysical gap modes). This agreement clearly supports our observations of strong electron transport through the subnanometric molecular junction of NMs. Additionally, we have noticed that the experimental resonance at the visible region becomes broader when the NM's size increases (Figure 6B). However, because this effect is not reproduced in the QCM calculations (Figure 6C), the broadening is not due to increased junction tunneling and dissipation but most likely due to extrinsic effects such as surface roughness or inhomogenmolecules at $t$ eous particle size.

Surface-enhancement Raman scattering (SERS) is a powerful probe of local field enhancement and charge transfer (CT) effects. ${ }^{11,70-73}$ Taking advantage of the presence of the 1,4-BDT molecules at the gap, we performed SERS measurement on the NMs to study the near-field enhancement and electron transfer in the molecular junction. Figure 4D shows the SERS spectra of $(10,10.7,25) \mathrm{nm}$ NMs in water, where the strong characteristic Raman bands of 1,4-BDT molecules at $729,1055,1551 \mathrm{~cm}^{-1}$ are observed when excited by $785 \mathrm{~nm}$ laser, and no significant bands are observed when excited by a $532 \mathrm{~nm}$ laser. The dramatic difference between the Raman intensities of the interior 1,4-BDT molecules excited by 785 and $532 \mathrm{~nm}$ lasers cannot be explained by as due to their associated near-field enhancements because the QCM-calculated electric field intensities in the junction at 532 and $785 \mathrm{~nm}$ are almost identical and very weak (Figure 4C). We hypothesize that the electron transport associated with the CT is the main enhancement mechanism that determines the SERS signal in the $(10,10.7,25)$ NMs system.
For further understanding of the effects of molecular transport, we have performed experiments also for 4-methylbenezenethiol (4-MBT) molecules, which have a very similar molecular structure to 1,4-BDT molecules. However, 4-MBT lacks the second thiol group compared to 1,4-BDT and therefore cannot establish a conductive link between the core and shell. Figure 7

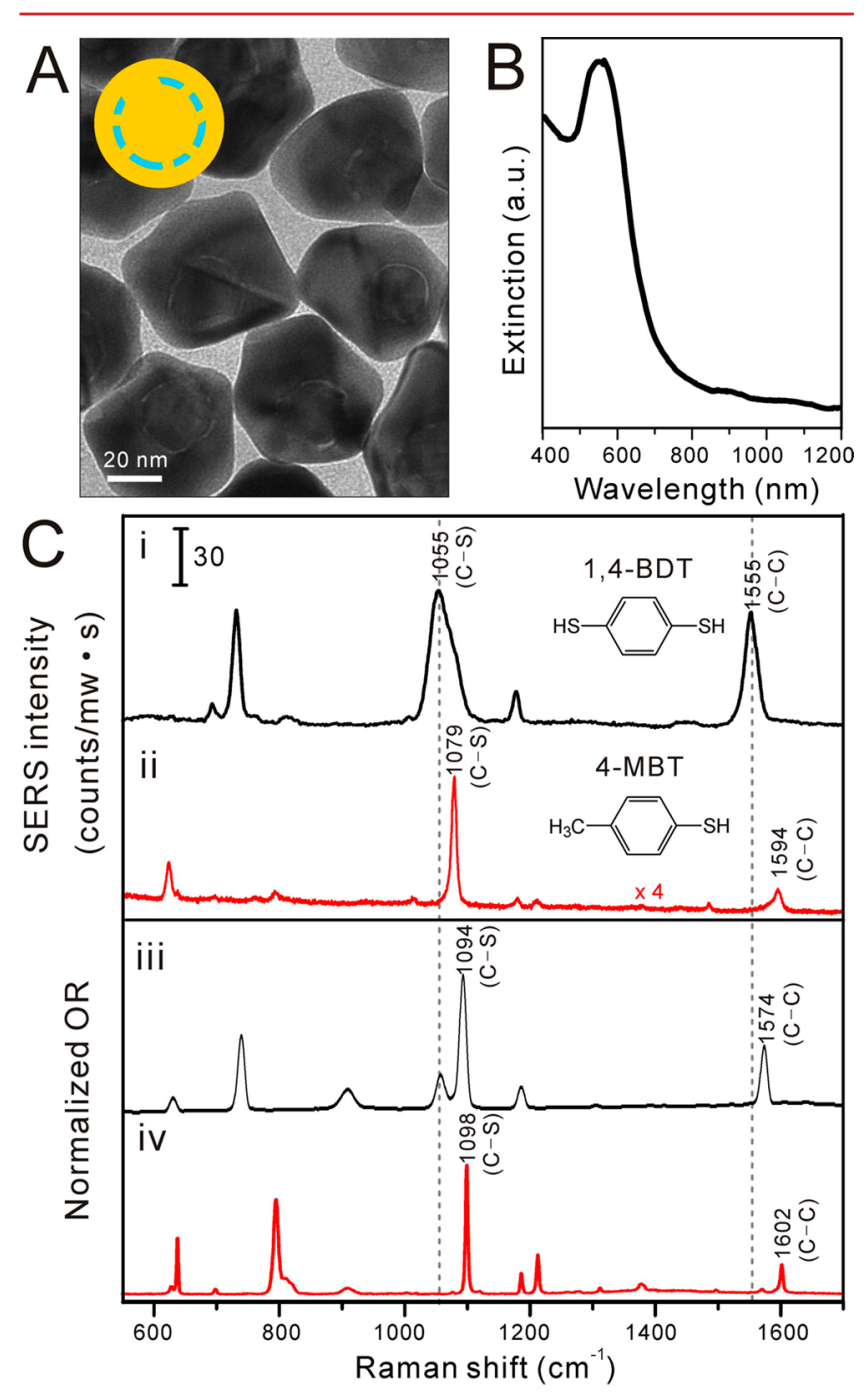

Figure 7. (A) TEM image and (B) extinction spectrum of 4-MBT embedded NMs. The inset in A denotes the inhomogeneous covering of the molecules at the NM gap. (C) SERS spectra of (i) 1,4-BDT and (ii) 4-MBT embedded NMs, and ordinary Raman (OR) spectra of (iii) 1,4BDT and (iv) 4-MBT solid.

summarizes and compares the results for 1,4-BDT and 4-MBT embedded NMs. First, we have only obtained 4-MBT embedded $\mathrm{NMs}$ with incomplete gaps (Figure 7A) even when full molecular coverage on the core is employed. Many metal bridges between the core and shell are still observed in that case. This is probably due to the presence of only one single thiol group in 4-MBT, which cannot form covalent bonds to Au at both ends. We have not observed the gap mode either in the long wavelength region of the extinction spectrum of 4-MBT embedded NMs, which can be attributed to the short circuiting caused by the current through the established metal bridges (Figure 7B).

For Raman measurements, we have observed three effects that demonstrate the CT effect only in the 1,4-BDT NMs instead of 4-MBT NMs: (1) Redshift of Raman bands. In our SERS 
measurements of 1,4-BDT NMs (i in Figure 7C), the $\mathrm{C}-\mathrm{S}$ ring breathing $\left(a_{1}\right)$ mode and the $C-C$ ring stretching $\left(a_{1}\right)$ mode both show a large redshift (shift to a lower wavenumber) of 39 $\mathrm{cm}^{-1}$ (from 1094 to $1055 \mathrm{~cm}^{-1}$ ) and $19 \mathrm{~cm}^{-1}$ (from 1574 to 1555 $\left.\mathrm{cm}^{-1}\right)$, respectively, compared to ordinary Raman (OR) spectrum of 1,4-BDT powder (iii in Figure 7C). Here, a silicon substrate was applied for signal calibration during all the Raman measurements. Our measurements have been carried out many times on three different Raman spectrometers (Bruker Optics Senterra R200-L Raman spectrophotometer, Renishaw Inviareflex Raman spectrophotometer, and Horiba Scientific LabRAM HR Evolution Raman spectrophotometer). Similar and consistent results were obtained confirming that the redshift of Raman bands was not induced by the instability or variation of the Raman system. In contrast to the case of 1,4-BDT, the redshift for 4-MBT NMs is much weaker. An examination of the SERS and OR spectra of 4-MBT gives a shift of $19 \mathrm{~cm}^{-1}$ (from 1098 to $1079 \mathrm{~cm}^{-1}$ ) and $8 \mathrm{~cm}^{-1}$ (from 1602 to $1594 \mathrm{~cm}^{-1}$ ) for the $\mathrm{C}-\mathrm{S}$ ring breathing mode and $\mathrm{C}-\mathrm{C}$ ring stretching mode, respectively (ii and iv in Figure $7 \mathrm{C}$ ). We attribute this redshift to the CT effect, which is related to the electron transfer between the lowest unoccupied molecular orbital/hightest occupied molecular orbital (LUMO/HOMO) of the adsorbed molecules and the Fermi level of Au. This effect would not only enhance the intensity of Raman scattering but also change the peak positions of specific Raman bands. ${ }^{74}$ According to the predictions from vibronic coupling theory, the CT effect is allowed for the $a_{1}$ bands, ${ }^{75,76}$ and a non-negligible CT activity of the $\mathrm{a}_{1}$ mode has also been reported in previous studies. ${ }^{77}$ When 1,4-BDT is adsorbed on the Au surface through the two thiol groups, the electrons are transferred from the HOMO of the molecule to the unfilled state of $\mathrm{Au}$, as well as from the occupied $\mathrm{Au}$ state to the LUMO of the molecule. This strong CT results in a decrease in the bond strength of $\mathrm{C}-\mathrm{S}$ and a decrease of electron cloud density around the benzene ring, which leads to the large redshift of the Raman band. ${ }^{73}$ However, a much weaker CT effect in 4MBT leads to only a small redshift of Raman bands. (2) Broadening of Raman bands. The time-averaged Raman line width of 1,4-BDT solids is significantly narrower than that of the 1,4-BDT in the molecular junction for the $\mathrm{C}-\mathrm{S}$ ring breathing $\left(a_{1}\right)$ mode and $C-C$ ring stretching $\left(a_{1}\right)$ mode. On average, the full width at half-maximum ( $\mathrm{fwhm}$ ) of the bands are $13 \mathrm{~cm}^{-1}$ (at $1094 \mathrm{~cm}^{-1}$ ) and $12.5 \mathrm{~cm}^{-1}$ (at $1574 \mathrm{~cm}^{-1}$ ) in 1,4-BDT powder and become to $42 \mathrm{~cm}^{-1}$ (at $1055 \mathrm{~cm}^{-1}$ ) and $24 \mathrm{~cm}^{-1}$ (at 1555 $\mathrm{cm}^{-1}$ ) in 1,4-BDT NMs. This is also consistent with the spatial heterogeneities of the CT effect. ${ }^{72}$ The junctions are made of $\mathrm{Au}$ core and shell with various crystalline facets and atom-sized defects, which may induce a fluctuation of local Fermi energies of metals. Next, there were numerous 1,4-BDT molecules with different orientations and conformations located in the gap, which may have various CT effects according to the surface selection rule. Numerous 1,4-BDT molecules with heterogeneous CT active states contribute to the different band-shifts, therefore resulting in the broadening of SERS bands for 1,4-BDT NMs. However, the broadening of bands becomes not obvious in the 4-MBT NMs due to a weaker CT effect. (3) Enhancement of Raman bands. By normalizing the laser power, integration time, and molecular concentration, the SERS intensity of 1,4-BDT NMs is 2 and 15 times stronger than that of 4-MBT NMs in terms of the $\mathrm{C}-\mathrm{S}$ Raman band at $1055 \mathrm{~cm}^{-1}$ and $\mathrm{C}-\mathrm{C}$ at 1555 $\mathrm{cm}^{-1}$, respectively (Figure $7 \mathrm{C}$ ). The stronger SERS intensity of 1,4-BDT NMs compared to 4-MBT NMs is thus most likely attributed to the stronger $\mathrm{CT}$ effect, since the electromagnetic enhancement in 1,4-BDT and 4-MBT NMs are both rather weak due to the establishment of the molecular or metal bridges.

In summary, there are two possibilities of electron transport across the gaps of NMs: either molecular conduction or through established metallic bridges. The vanishing of the gap mode, the strong CT effect, and the absence of metallic bridges as inferred from the TEM examination of a large amount of particles all suggest electron transport through the molecular junction in 1,4BDT NMs. Furthermore, for a situation where no electron transport occurs via the subnanometric molecular junction, for example, due to small molecular conductance, we have theoretically found that a small number of metal bridges (e.g., one or two) do not produce the quenching of the cavity mode in the extinction spectrum (Supporting Information Figure S3). Only when the number of metallic bridges increases substantially, a short-circuiting situation is reached that determines the optical spectrum. This corroborates that the effect of gap mode quenching that we identify in 1,4-BDT NMs is is due to the electron transport through the molecular junction. In contrast, in 4-MBT NMs the TEM characterization and rather weak CT effect implies the electron transport to occur through the metal bridges.

Currently, the advances of the field of quantum plasmonics are limited by the development of suitable techniques for fabrication of subnanometer nanocavities. Our NM system offers a number of advantages compared to other systems: (i) large crosssectional area $\left(1000-5000 \mathrm{~nm}^{2}\right)$ of the molecular transporting junction, maximizing the number of transporting events across the gaps; (ii) the formation of a metal-molecule-metal junction, increasing the transporting rate through conducting molecules compared to vacuum or other types of dielectric spacers; (iii) solution-based synthesis, providing a scalable approach for mass production. Extensions and optimization of our approach are likely to further enhance these advantages. Although the 1,4-BDT spacing layer exhibits a remarkable uniformity, the optical response in this work was measured from ensembles of NMs in solution. Further optical characterization, for example, dark-field scattering spectrum and SERS measurements on individual NMs, may offer additional insight into quantum plasmonics phenomena.

In conclusion, we have experimentally and theoretically investigated the optical responses of $\mathrm{Au}$ nanomatryoshkas with core-shell gaps ranging from $100 \mathrm{~nm}$ to the subnanometer regime. As the gap is decreased, the coupling between core and shell plasmons increases and results in increasingly strong hybridization. For subnanometer size gaps, quantum effects such as electron transporting via molecular junctions between the core and the shell become important, resulting in the charge transfer screening of one of the hybridized modes at the gap. Our theoretical QCM simulations successfully reproduce the measured far-field spectra and are further utilized for the probing of near-field enhancement in the molecular junction. The emergence of charge transfer is also consistent with SERS measurements of conductive 1,4-BDT and nonconductive 4MBT molecules in the junction. Our scalable bottom-up approach for fabrication of nanoparticles exhibiting strong quantum plasmonics effects may pave the path for the development of novel plasmonic devices based on quantum effects. The quantum properties exploited in this work can provide a new insight on the ongoing developing strategies searching for optimal plasmonic SERS substrates with narrow junction gaps or sharp protrusions and could also be further utilized to access molecular conductance in nanojunctions. 


\section{ASSOCIATED CONTENT}

\section{S Supporting Information}

The Supporting Information is available free of charge on the ACS Publications website at DOI: 10.1021/acs.nanolett.5b02931.

Additional figures, materials, synthesis of $(25,125,150)$ $\mathrm{nm}$ nanomatryoshka particles, synthesis of 1,4-BDT embedded nanomatryoshka particles, and additional references. (PDF)

\section{AUTHOR INFORMATION}

\section{Corresponding Authors}

*E-mail: (J.A.) aizpurua@ehu.eus.

*E-mail: (P.N.) nordland@rice.edu.

*E-mail: (J.Y.) yejian78@sjtu.edu.cn.

\section{Author Contributions}

L.L. and M.Z. contributed equally to this work.

Notes

The authors declare no competing financial interest.

\section{ACKNOWLEDGMENTS}

We gratefully acknowledge the National Natural Science Foundation of China (No. 21375087 and No. 81571763), the Science and Technology Commission of Shanghai Municipality (No. 13ZR1422100) and Shanghai Jiao Tong University (No. YG2014MS53 and SJTU-KUL Bilateral Program) for their financial support. M.Z. and J.A. acknowledge financial support from Project "Plasmogap" (FIS2013-41184-P) of the Spanish Ministry of Economy and Competitiveness (MINECO), and project Etortek Nanogune'14 of the Department of Industry of the Basque Government. M.Z. acknowledges financial support from the Departamento Administrativo de Ciencia, Tecnologia e Innovación-COLCIENCIAS and Facultad de Ciencias from Universidad de los Andes. P.N. Acknowledges support from the Robert A. Welch Foundation (C-1222) and the ARO-Muri (W911NF-12-1-0407).

\section{REFERENCES}

(1) Atwater, H. A.; Polman, A. Nat. Mater. 2010, 9, 205-213.

(2) Christopher, P.; Xin, H.; Linic, S. Nat. Chem. 2011, 3, 467-472.

(3) Linden, S.; Enkrich, C.; Wegener, M.; Zhou, J.; Koschny, T.; Soukoulis, C. M. Science 2004, 306, 1351-1353.

(4) Shalaev, V. M. Nat. Photonics 2007, 1, 41-48.

(5) Zijlstra, P.; Chon, J. W.; Gu, M. Nature 2009, 459, 410-413.

(6) Maier, S. A.; Kik, P. G.; Atwater, H. A.; Meltzer, S.; Harel, E.; Koel, B. E.; Requicha, A. A. Nat. Mater. 2003, 2, 229-232.

(7) Anker, J. N.; Hall, W. P.; Lyandres, O.; Shah, N. C.; Zhao, J.; Van Duyne, R. P. Nat. Mater. 2008, 7, 442-453.

(8) Kravets, V. G.; Schedin, F.; Jalil, R.; Britnell, L.; Gorbachev, R. V.; Ansell, D.; Thackray, B.; Novoselov, K. S.; Geim, A. K.; Kabashin, A. V.; Grigorenko, A. N. Nat. Mater. 2013, 12, 304-309.

(9) Khoury, C. G.; Norton, S. J.; Vo-Dinh, T. ACS Nano 2009, 3, 2776-2788.

(10) Ye, J.; Van Dorpe, P. Nanoscale 2012, 4, 7205-7211.

(11) Ye, J.; Wen, F. F.; Sobhani, H.; Lassiter, J. B.; Van Dorpe, P.; Nordlander, P.; Halas, N. J. Nano Lett. 2012, 12, 1660-1667.

(12) Wen, F.; Ye, J.; Liu, N.; Van Dorpe, P.; Nordlander, P.; Halas, N. J. Nano Lett. 2012, 12, 5020-5026.

(13) Prodan, E.; Radloff, C.; Halas, N.; Nordlander, P. Science 2003, 302, 419-422.

(14) Bardhan, R.; Mukherjee, S.; Mirin, N. A.; Levit, S. D.; Nordlander, P.; Halas, N. J. J. Phys. Chem. C 2010, 114, 7378-7383.

(15) Ayala-Orozco, C.; Liu, J. G.; Knight, M. W.; Wang, Y.; Day, J. K.; Nordlander, P.; Halas, N. J. Nano Lett. 2014, 14, 2926-2933.
(16) Shen, W.; Lin, X.; Jiang, C.; Li, C.; Lin, H.; Huang, J.; Wang, S.; Liu, G.; Yan, X.; Zhong, Q. Angew. Chem., Int. Ed. 2015, 54, 7308-7312.

(17) Hong, S.; Acapulco, J. A., Jr; Jang, H. Y.; Park, S. Chem. Mater. 2014, 26, 3618-3623.

(18) Song, J.; Duan, B.; Wang, C.; Zhou, J.; Pu, L.; Fang, Z.; Wang, P.; Lim, T. T.; Duan, H. J. Am. Chem. Soc. 2014, 136, 6838-6841.

(19) Feng, Y.; Wang, Y.; Wang, H.; Chen, T.; Tay, Y. Y.; Yao, L.; Yan, Q.; Li, S.; Chen, H. Small 2012, 8, 246-251.

(20) Mukherjee, S.; Sobhani, H.; Lassiter, J. B.; Bardhan, R.; Nordlander, P.; Halas, N. J. Nano Lett. 2010, 10, 2694-2701.

(21) Hu, Y.; Noelck, S. J.; Drezek, R. A. ACS Nano 2010, 4, 15211528.

(22) Kulkarni, V.; Prodan, E.; Nordlander, P. Nano Lett. 2013, 13, $5873-5879$.

(23) Zapata, M.; Beltran, A. S. C.; Borisov, A. G.; Aizpurua, J. Opt. Express 2015, 23, 8134-8149.

(24) Kang, J. W.; So, P. T.; Dasari, R. R.; Lim, D.-K. Nano Lett. 2015, $15,1766-1772$

(25) Zhao, B.; Shen, J.; Chen, S.; Wang, D.; Li, F.; Mathur, S.; Song, S.; Fan, C. Chem. Sci. 2014, 5, 4460-4466.

(26) Scholl, J. A.; García-Etxarri, A.; Koh, A. L.; Dionne, J. A. Nano Lett. 2013, 13, 564-569.

(27) Tan, S. F.; Wu, L.; Yang, J. K.; Bai, P.; Bosman, M.; Nijhuis, C. A. Science 2014, 343, 1496-1499.

(28) Savage, K. J.; Hawkeye, M. M.; Esteban, R.; Borisov, A. G.; Aizpurua, J.; Baumberg, J. J. Nature 2012, 491, 574-577.

(29) Tame, M.; McEnery, K.; Özdemir, Ş.; Lee, J.; Maier, S.; Kim, M. Nat. Phys. 2013, 9, 329-340.

(30) Duan, H.; Fernández-Domínguez, A. I.; Bosman, M.; Maier, S. A.; Yang, J. K. Nano Lett. 2012, 12, 1683-1689.

(31) Kern, J.; Großmann, S.; Tarakina, N. V.; Häckel, T.; Emmerling, M.; Kamp, M.; Huang, J.-S.; Biagioni, P.; Prangsma, J. C.; Hecht, B. Nano Lett. 2012, 12, 5504-5509.

(32) Esteban, R.; Borisov, A. G.; Nordlander, P.; Aizpurua, J. Nat. Commun. 2012, 3, 825.

(33) Wu, L.; Duan, H.; Bai, P.; Bosman, M.; Yang, J. K.; Li, E. ACS Nano 2013, 7, 707-716.

(34) Marinica, D.; Kazansky, A.; Nordlander, P.; Aizpurua, J.; Borisov, A. G. Nano Lett. 2012, 12, 1333-1339.

(35) Zuloaga, J.; Prodan, E.; Nordlander, P. Nano Lett. 2009, 9, 887891.

(36) Lim, D.-K.; Jeon, K.-S.; Hwang, J.-H.; Kim, H.; Kwon, S.; Suh, Y. D.; Nam, J.-M. Nat. Nanotechnol. 2011, 6, 452-460.

(37) Gandra, N.; Singamaneni, S. Adv. Mater. 2013, 25, 1022-1027.

(38) Esteban, R.; Zugarramurdi, A.; Zhang, P.; Nordlander, P.; GarciaVidal, F. J.; Borisov, A. G.; Aizpurua, J. Faraday Discuss. 2015, 178, 151183.

(39) Johnson, P. B.; Christy, R.-W. Phys. Rev. B 1972, 6, 4370-4379.

(40) Palik, E. D. Handbook of Optical Constants of Solids; Academic Press: Orlando, FL, 1985.

(41) Walczak, M. M.; Chung, C.; Stole, S. M.; Widrig, C. A.; Porter, M. D. J. Am. Chem. Soc. 1991, 113, 2370-2378.

(42) Ozbay, E. Science 2006, 311, 189-193.

(43) Ye, J.; Van Dorpe, P.; Van Roy, W.; Borghs, G.; Maes, G. Langmuir 2009, 25, 1822-1827.

(44) Oldenburg, S. J.; Averitt, R. D.; Westcott, S. L.; Halas, N. J. Chem. Phys. Lett. 1998, 288, 243-247.

(45) Nehl, C. L.; Grady, N. K.; Goodrich, G. P.; Tam, F.; Halas, N. J.; Hafner, J. H. Nano Lett. 2004, 4, 2355-2359.

(46) Ye, J.; de Broek, B.; De Palma, R.; Libaers, W.; Clays, K.; Van Roy, W.; Borghs, G.; Maes, G. Colloids Surf., A 2008, 322, 225-233.

(47) Li, J. F.; Huang, Y. F.; Ding, Y.; Yang, Z. L.; Li, S. B.; Zhou, X. S.; Fan, F. R.; Zhang, W.; Zhou, Z. Y.; Wu, D. Y. Nature 2010, 464, 392395.

(48) Bain, C. D.; Troughton, E. B.; Tao, Y. T.; Evall, J.; Whitesides, G. M.; Nuzzo, R. G. J. Am. Chem. Soc. 1989, 111, 321-335.

(49) Joo, S. W.; Han, S. W.; Kim, K. J. Colloid Interface Sci. 2001, 240, 391-399. 
(50) Pontes, R. B.; Novaes, F. D.; Fazzio, A.; da Silva, A. J. J. Am. Chem. Soc. 2006, 128, 8996-8997.

(51) Reed, M. A.; Zhou, C.; Muller, C.; Burgin, T.; Tour, J. Science 1997, 278, 252-254.

(52) Nitzan, A.; Ratner, M. A. Science 2003, 300, 1384-1389.

(53) Tao, N. Nat. Nanotechnol. 2006, 1, 173-181.

(54) Reddy, P.; Jang, S.-Y.; Segalman, R. A.; Majumdar, A. Science 2007, 315, 1568-1571.

(55) Xiao, X.; Xu, B.; Tao, N. J. Nano Lett. 2004, 4, 267-271.

(56) Tsutsui, M.; Taniguchi, M.; Kawai, T. Nano Lett. 2009, 9, 24332439.

(57) Strange, M.; Rostgaard, C.; Häkkinen, H.; Thygesen, K. S. Phys. Rev. B: Condens. Matter Mater. Phys. 2011, 83, 115108.

(58) Pontes, R. B.; Rocha, A. R.; Sanvito, S.; Fazzio, A.; da Silva, A. J. R. ACS Nano 2011, 5, 795-804.

(59) Venkataraman, L.; Klare, J. E.; Nuckolls, C.; Hybertsen, M. S.; Steigerwald, M. L. Nature 2006, 442, 904-907.

(60) Kiguchi, M.; Nakamura, H.; Takahashi, Y.; Takahashi, T.; Ohto, T. J. Phys. Chem. C 2010, 114, 22254-22261.

(61) Kim, Y.; Pietsch, T.; Erbe, A.; Belzig, W.; Scheer, E. Nano Lett. 2011, 11, 3734-3738.

(62) Bürkle, M.; Viljas, J. K.; Vonlanthen, D.; Mishchenko, A.; Schön, G.; Mayor, M.; Wandlowski, T.; Pauly, F. Phys. Rev. B: Condens. Matter Mater. Phys. 2012, 85, 075417.

(63) Benz, F.; Tserkezis, C.; Herrmann, L. O.; de Nijs, B.; Sanders, A.; Sigle, D. O.; Pukenas, L.; Evans, S. D.; Aizpurua, J.; Baumberg, J. J. Nano Lett. 2015, 15, 669-674.

(64) Pérez-González, O.; Zabala, N.; Borisov, A.; Halas, N.; Nordlander, P.; Aizpurua, J. Nano Lett. 2010, 10, 3090-3095.

(65) Love, J. C.; Estroff, L. A.; Kriebel, J. K.; Nuzzo, R. G.; Whitesides, G. M. Chem. Rev. 2005, 105, 1103-1170.

(66) García de Abajo, F. J.; Howie, A. Phys. Rev. B: Condens. Matter Mater. Phys. 2002, 65, 115418.

(67) Baer, R.; Seideman, T.; Ilani, S.; Neuhauser, D. J. Chem. Phys. 2004, 120, 3387-3396.

(68) Keller, A.; Atabek, O.; Ratner, M.; Mujica, V. J. Phys. B: At., Mol. Opt. Phys. 2002, 35, 4981-4988.

(69) Song, P.; Meng, S.; Nordlander, P.; Gao, S. Phys. Rev. B: Condens. Matter Mater. Phys. 2012, 86, 121410.

(70) Zhu, W.; Crozier, K. B. Nat. Commun. 2014, 5, 5228.

(71) Ye, J.; Chen, C.; Lagae, L.; Maes, G.; Borghs, G.; Van Dorpe, P. Phys. Chem. Chem. Phys. 2010, 12, 11222-11224.

(72) Park, W.-H.; Kim, Z. H. Nano Lett. 2010, 10, 4040-4048.

(73) Matsuhita, R.; Horikawa, M.; Naitoh, Y.; Nakamura, H.; Kiguchi, M. J. Phys. Chem. C 2013, 117, 1791-1795.

(74) Tong, L.; Zhu, T.; Liu, Z. Chem. Soc. Rev. 2011, 40, 1296-1304.

(75) Cho, S. H.; Han, H. S.; Jang, D.-J.; Kim, K.; Kim, M. S. J. Phys. Chem. 1995, 99, 10594-10599.

(76) Osawa, M.; Matsuda, N.; Yoshii, K.; Uchida, I. J. Phys. Chem. 1994, 98, $12702-12707$.

(77) Chenal, C.; Birke, R. L.; Lombardi, J. R. ChemPhysChem 2008, 9, $1617-1623$. 